\title{
Pour une approche des routines discursives dans les écrits professionnels
}

\author{
Née, Emilie, Sitri, Frédérique et Veniard, Marie \\ Université Paris Est Créteil et Céditec, Université Paris Ouest Nanterre et Modyco, \\ Université Paris Descartes et EDA \\ emilie.nee@u-pec.fr, frederique.sitri@u-paris10.fr, marie.veniard@parisdescartes.fr
}

Dans cette contribution, nous souhaitons proposer une analyse de l'écrit professionnel en termes de routines discursives, phénomène que nous situons à l'articulation entre des déterminations discursives, des phénomènes de figement et le processus rédactionnel. Sur le plan méthodologique, cela passe par l'exploitation de figements de niveau textuel et par la mise au jour d'enchaînements phraséologiques de niveau sémantico-pragmatique articulant plusieurs segments figés ou patterns. Ce faisant, nous montrerons ce que l'étude de la phraséologie peut apporter à l'analyse de discours dans la caractérisation des genres et des sphères d'activité, en donnant une assise matérielle et un contenu notionnel à la catégorie de « routines » souvent invoquée pour caractériser un discours ou un genre (Charaudeau 2001, Maingueneau 2004, Krieg-Planque 2008, etc.).

Cette communication s'inscrit dans le projet ANR Ecriture (Resp. G. Cislaru) et porte sur des rapports éducatifs rédigés par des éducateurs spécialisés dans le champ de la protection de l'enfance. L'un des objectifs de ce projet est l'étude du processus d'écriture via un corpus offrant un triple regard sur les données: états finaux des textes, suivi des modifications dans les états successifs et suivi des modifications en temps réel. Les explorations textométriques développées ici sont menées sur le seul corpus des états finaux (voir Née, MacMurray et Fleury 2012).

Pour décrire ces écrits, nous ne pouvions nous limiter à la recension de collocations spécialisées (famille d'accueil, poser un acte, pour exécuter un acte), entrée trop restreinte à nos yeux qui ne permet pas de prendre en compte les enjeux pragmatiques de ces textes, en relation avec le genre dont ils relèvent. Nous avons tenté de saisir d'autres types de figements, plus longs, plus diversifiés, en prenant appui sur des travaux qui mettent en avant le rattachement de séquences plus ou moins figées à des déterminations d'ordre supérieur, à savoir le discours d'une part et le texte d'autre part. Pour les déterminations discursives, nous nous référons à la fois aux travaux du laboratoire de lexicologie politique de SaintCloud sur les Congrès syndicaux (Fiala 1987, Fiala et alii 1987) et aux travaux de Sonia Branca-Rosoff (1990 et 1997) sur les écrits des peu lettrés. Pour les déterminations textuelles, nous faisons référence à la catégorie du motif développée par Longrée, Luong et Mellet 2008, Longrée et Mellet 2013. Ce faisant, nous nous inscrivons dans les propositions d'extension du domaine de la phraséologie de la langue au discours présentées par Dominique Legallois et Agnès Tutin dans Langages 189 (2013). Parallèlement, nous nous sommes inspirées des travaux de la linguistique de corpus britannique qui développe des notions permettant d'articuler phraséologie locale et visée pragmatique globale (Sinclair 2004).

Ce type de questionnement n'est pas étranger à l'analyse de discours qui, dans ses débuts en tout cas, considère que la répétition permet de dégager le sens d'un discours (Maingueneau (1991: 77) par exemple citant des travaux en analyse du discours). Dans une autre direction, la statistique textuelle, quand elle s'occupe de discours politique, fait de la répétition la voie d'accès au figement. Ainsi, au départ, est convoquée une approche statistique de la phraséologie (versus lexicologique, selon une distinction communément partagée, voir Bolly 2011 ou Legallois et Tutin 2013). C'est la naissance, issue de la rencontre entre l'analyse de discours et la statistique textuelle, de la notion de segment répété (Salem 1987), qui permet de saisir les effets de «langue de bois » (Fiala 1987, Fiala et alii 1987). Cette conjonction disciplinaire a également beaucoup développé le recours aux cooccurrences dont le calcul remonte à Lafon 1981 (Lafon 1981, Heiden et Lafon 1998, Martinez 2003, Mayaffre 2008, etc.) pour étudier des réseaux d'associations récurrentes.

Dans la perspective développée par le Laboratoire de Saint Cloud, le figement vient de la répétition et différents niveaux de figement sont identifiés, correspondant à différentes « intuitions de locutionnalité » : 
les figements en langue, les figements textuels, repérables à leur fréquence élevée dans le texte ou le genre analysé, les figements "en usage ", relevant d'usages discursifs, de genres, et le semi-figement (dans Fiala et alii 1987, le congrès syndical, le discours de la CGT...) ${ }^{1}$. Nous considérons, à la suite de ces travaux, que la prise en compte de ces différents niveaux de détermination du discours fournit de fait un horizon interprétatif aux formes figées. Tout en nous situant dans cette tradition, nous intégrons également les travaux sur la phraséologie qui se sont penchés récemment sur l'articulation entre phraséologie et genre ou entre phraséologie et sphère d'activité (Poudat 2006, Tutin 2007, Bolly 2011).

Enfin, il est ici utile de revenir sur un élément important de notre réflexion, qui nous a amenées à poser la problématique des « routines » discursives (et non seulement celle de la «phraséologie » d'un [genre de] discours). Notre réflexion sur le figement a en effet pris un tournant dès lors que nous avons travaillé sur l'activité d'écriture dans une perspective génétique et non plus seulement sur une production écrite « finie ». À l'analyse de la somme des versions finales de rapports éducatifs, s'est adjointe l'étude des brouillons, en l'occurrence des différents états de rédaction d'un même dossier. La problématique même des «routines discursives » est née de la conjonction de ces deux angles de vue sur les mêmes écrits : la perspective génétique permet de suivre l'apparition à un moment du texte des séquences identifiées comme plus ou moins figées et les modifications qui les affectent ou non.

Nos travaux ont concerné jusqu'à présent des entrées lexicales (Veniard 2008, Née, Mac Murray et Fleury 2011), les temps, la Représentation du Discours Autre (Sitri 2008). Cependant, le sentiment diffus d'une forme de routine dans ces écrits, sans qu'il s'agisse réellement de figement (en dehors d'unités polylexicales qui relèvent de la terminologie) nous est venu peu à peu. Il s'est affirmé lorsque les éducateurs, lors d'une séance de restitution, nous ont demandé s'ils utilisaient de la langue de bois. La perspective phraséologique nous a ainsi permis de regrouper des observables épars.

\section{Corpus, catégories de la statistique textuelle et outils exploratoires}

Les données sur lesquelles nous avons travaillées sont de deux sortes. D'une part, 22 rapports éducatifs dans leur version finale telle que les travailleurs sociaux nous les ont envoyées. D'autre part, les différents états de rédaction de chaque rapport (entre 2 et 41 états selon les dossiers), chaque état correspondant à une version numérique enregistrée par le travailleur social sur son propre ordinateur toutes les $15 \mathrm{mn}$. Les explorations textométriques dont il sera ici question ont essentiellement porté sur le corpus d'états finaux. En revanche l'alignement des états permet de suivre les modifications.

Nous avons utilisé un logiciel de textométrie, Le Trameur, conçu par Serge Fleury (voir Fleury 2007).

L'analyse des données s'est effectuée à partir des catégories de la statistique textuelle et des différents outils exploratoires suivants : 


\begin{tabular}{|c|c|c|}
\hline $\begin{array}{l}\text { Catégories et } \\
\text { méthodes de la } \\
\text { statistique textuelle }\end{array}$ & $\begin{array}{l}\text { Outil } \\
\text { exploratoire (Le } \\
\text { Trameur) }\end{array}$ & Rappel définition/algorithme \\
\hline $\begin{array}{l}\text { Fréquences (relatives } \\
\text { et absolues), } \\
\text { ventilations (dossiers, } \\
\text { états) }\end{array}$ & $\begin{array}{l}\text { dictionnaires des } \\
\text { formes, ventilation }\end{array}$ & $\begin{array}{l}\text { La fréquence absolue d'une unité dans un corpus } \mathrm{T} \text { ou } \\
\text { une partie } t \text { correspond à la fréquence maximale de cette } \\
\text { unité dans } \mathrm{T} \text { ou } \mathrm{t} \text {; } \\
\text { la fréquence relative d'une unité dans un corpus } \mathrm{T} \text { ou } \\
\text { une partie t correspond à la fréquence de cette unité dans } \\
\mathrm{T} \text { ou t rapportée à la taille de T ou } \mathbf{t} \text { (= Freq unité /t ou } \\
\text { T) }\end{array}$ \\
\hline Concordances & $\begin{array}{l}\text { le concordancier } \\
\text { simple } \\
\text { la fonctionnalité } \\
\text { patron }\end{array}$ & $\begin{array}{l}\text { construction (syntaxique/morpho/sémantique) autour } \\
\text { d'une forme-pôle }\end{array}$ \\
\hline Segments répétés & $\begin{array}{l}\text { dictionnaire des } \\
\text { segments répétés }\end{array}$ & $\begin{array}{l}\text { une suite de formes non séparées par une ponctuation dont } \\
\text { la fréquence est égale ou supérieure à deux dans un corpus }\end{array}$ \\
\hline $\begin{array}{l}\text { Calcul des } \\
\text { cooccurrences }\end{array}$ & cooccurrences & 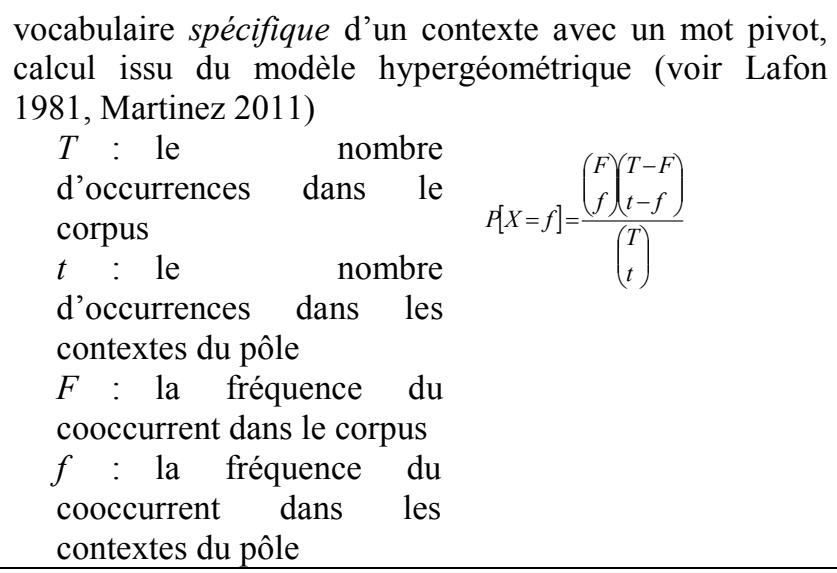 \\
\hline
\end{tabular}

Tableau 1. Les catégories descriptives de la statistique textuelle utilisées comme outils exploratoires

Nos outils exploratoires, segments répétés (SR) et cooccurrences (seuillées), ont présenté, par rapport à notre objectif, des limites que nous avons dépassées en trouvant dans le champ de la phraséologie des catégories descriptives plus opérationnelles. De fait, il nous fallait passer des SR et des cooccurrences à des unités pertinentes aux plans syntagmatique (ce qui n'est pas le cas des cooccurrences et qui n'est que parfois le cas des SR) et sémantico-discursif.

De plus, en même temps que nous avons mis en évidence différentes séquences figées, s'est posée la question des propriétés et du classement de ces unités, questionnement déjà à l'œuvre dans les approches discursives de la phraséologie (voir Fiala 1987).

\section{Les formes mises au jour : des patrons}

Nous considérons ainsi que les unités phraséologiques peuvent dépasser le cadre du syntagme et sont de nature multidimensionnelle : elles peuvent être constituées d'unités hétérogènes sur le plan formel d'une part et d'autre part être mises en relation avec des fonctions ou des déterminations textuelles ou 
discursives. Nous empruntons à la linguistique de corpus et au traitement automatique des langues le terme de «patron » que nous définissons de la façon suivante :

Définition des patrons : moule syntaxique avec une combinatoire lexicale plus ou moins restreinte. Les patrons peuvent être de l'ordre du syntagme ou de la proposition (patron syntaxique), voire avoir un empan inter-propositionnel (patron séquentiel). Ils peuvent comporter des places qui ne sont pas toujours toutes actualisées. Nous posons l'existence d'un continuum entre des séquences très figées et d'autres qui le sont moins. Le moindre degré de figement est représenté par des associations notionnelles.

D'une certaine manière, la notion de patron telle que nous l'entendons se rapproche de la notion de «motif » proposée par D. Longrée et $\mathrm{S}$. Mellet, cette dernière étant définie comme

«une nouvelle unité textuelle, qui intégrant formes graphiques, lemmes, catégories grammaticales, patrons syntaxiques et, éventuellement, schèmes métriques ou prosodiques, permette de traiter à la fois l'imbrication hiérarchique de ces différents niveaux linguistiques, leur association syntagmatique dans la chaîne linéaire des énoncés et leur récurrence structurante au niveau macro-textuel » (Mellet et Longrée 2012 : 717).

Longrée et Mellet (2012) soulignent l'importance de la fonction textuelle des motifs : « Ainsi un motif a une fonction textuelle et discursive, à courte et à longue portée » (Mellet et Longrée 2012 : 717), par exemple des fonctions cohésive, résomptive, de structuration temporelle de la narration, de progression de l'intrigue, etc. Toutefois, dans les exemples donnés par les auteurs, les motifs ont majoritairement des fonctions textuelles, et non discursives telles que nous l'entendons du moins dans une perspective d'analyse du discours, c'est-à-dire la prise en compte des déterminations discursives et interdiscursives en relation avec la construction d'une interprétation (voir section 3 ).

Nous avons choisi de sélectionner un certain nombre de formes dont la plupart figure dans la liste des fréquences et segments répétés.

\begin{tabular}{|c|c|c|}
\hline Patrons & Exemples & Remarques \\
\hline $\begin{array}{l}\text { Patron « sur le groupe » } \\
\text { sur le groupe } \rightarrow \text { patron : «sur }+ \\
(\text { det }+) N »\end{array}$ & $\begin{array}{l}\text { depuis qu'il est arrivé sur le groupe } \\
\text { Elle est devenue la jeune fille la plus âgée sur le } \\
\text { groupe } \\
\text { Du fait de la grippe a, elle était chez sa mère } \\
\text { lors de la soirée de noël sur le groupe } \\
\text { Les départs en famille et les retours sur le } \\
\text { groupe sont généralement chaleureux. } \\
\text { Sa place sur le groupe a évolué. } \\
\text { Pour s'autoriser à mieux s'installer sur le } \\
\text { groupe } \\
\text { Impliquée dans de nombreuses histoires sur le } \\
\text { groupe } \\
\text { En raison d'un vol de pantalon sur le groupe }\end{array}$ & $\begin{array}{l}\text { Peu de variantes } \\
\text { combinatoires dans le corpus }\end{array}$ \\
\hline $\begin{array}{l}\text { Patron « être dans » } \\
\mathrm{X} \text { est dans }+[\operatorname{adj}]+\mathrm{N} \\
\text { tel que } \mathrm{N} \text { soit un } \mathrm{N} \text { prédicatif } \\
\text { abstrait, le plus souvent un }\end{array}$ & $\begin{array}{l}\text { il est dans la dissimulation de ses devoirs ou } \\
\text { mots à faire signer } \\
\text { est toujours dans une recherche d'intérêt de la } \\
\text { part de la famille d'accueil }\end{array}$ & \\
\hline
\end{tabular}




\begin{tabular}{|c|c|c|}
\hline déverbal ou un dérivé d'adjectif & $\begin{array}{l}\text { est dans une demande de pouvoir se rendre } \\
\text { B est dans l'évitement du conflit } \\
\text { il peut être ensuite dans la réparation } \\
\text { est dans un conflit de loyauté très important } \\
\text { reste dans une grande difficulté } \\
\text { il reste encore dans le besoin de satisfaction } \\
\text { immédiate } \\
\text { la confusion dans laquelle A est dans sa propre } \\
\text { histoire }\end{array}$ & \\
\hline $\begin{array}{l}\text { Patron « être en » } \\
\mathrm{X} \text { est en }+[\operatorname{adj}]+\mathrm{N} \\
\text { tel que } \mathrm{N} \text { soit un } \mathrm{N} \text { prédicatif } \\
\text { abstrait, le plus souvent un } \\
\text { déverbal ou un dérivé d'adjectif }\end{array}$ & $\begin{array}{l}\text { Test en recherche affective de la part de sa } \\
\text { mère } \\
\text { Test en attente de gratification } \\
\text { Elle est en grande demande affective } \\
\text { M est en demande auprès du service } \\
\text { ne nous semble pas en capacité d'élaborer }\end{array}$ & \\
\hline $\begin{array}{l}\text { Patron « être en difficulté » } \\
\text { X est en difficulté pour + SINF }\end{array}$ & $\begin{array}{l}\text { madame est en grande difficulté pour se } \\
\text { mobiliser autour des besoins de ses enfants et } \\
\text { de l'exercice de son autorité parentale } \\
\text { Houria est en grande difficulté pour jouer } \\
\text { seule } \\
\text { elle est toujours en difficulté pour se } \\
\text { concentrer } \\
\text { elle apprécie les travaux manuels et n'est pas } \\
\text { en difficulté pour occuper ses temps libres } \\
\text { Madame est en grande difficulté pour se } \\
\text { mobiliser autour des besoins de ses enfants } \\
\text { Madame Vaillant est régulièrement en } \\
\text { difficulté pour évaluer si il y a nécessiter à } \\
\text { appeler } \\
\text { elle supporte toujours très difficilement la } \\
\text { frustration et est en difficulté pour canaliser } \\
\text { ses états d'énervement. }\end{array}$ & sous-type du précédent \\
\hline $\begin{array}{l}\text { patron « Pouvoir » } \\
{[\text { et }]+[\text { circonstant }]+\mathrm{X} \text { peut } / \mathrm{a} \text { pu }} \\
+[\text { adverbe/circonstant }]+[\text { nous }] \\
+ \text { Prédicat « difficile à dire ou à } \\
\text { faire » }\end{array}$ & $\begin{array}{l}\text { il a pu nous faire part de ses réticences } \\
\text { Il peut aujourd'hui partager certains moments } \\
\text { agréables avec les autres sans être envahi par } \\
\text { ses peurs }\end{array}$ & \\
\hline $\begin{array}{l}\text { patron « et peut » } \\
\text { PROP ET PEUT SINF }\end{array}$ & $\begin{array}{l}\text { - elle est autonome dans les jeux, et peut rester } \\
\text { un long moment à jouer seule dans sa chambre }\end{array}$ & sous-type du précédent \\
\hline
\end{tabular}




\begin{tabular}{|c|c|}
\hline & $\begin{array}{l}\text { - mais ses positions sont très fluctuantes et elle } \\
\text { peut changer d'avis suivant la personne avec } \\
\text { qui elle échange ou le moment. } \\
\text { - c'est pourquoi aujourd'hui il a évolué dans ses } \\
\text { réflexions et peut mesurer un peu plus } \\
\text { l'importance d'une telle formation pour son } \\
\text { avenir professionnel. }\end{array}$ \\
\hline $\begin{array}{l}\text { patron «Temporalité } \\
\text { modalité (capacité)» } \\
\text { ADV de temps (aspect } \\
\text { imperfectif, rupture avec le } \\
\text { passé) + prédicat exprimant la } \\
\text { capacité) }\end{array}$ & $\begin{array}{l}\text { pour autant, Didier démontre désormais } \\
\text { certaines capacités d'autonomie qu'il a } \\
\text { récemment acquises } \\
\text { plus ouverte aux autres qu'auparavant, moins } \\
\text { angoissée dans son rapport à autrui et } \\
\text { désormais plus capable de démarches } \\
\text { extérieures qu'autrefois } \\
\text { ainsi, elle peut maintenant prendre du recul } \\
\text { face aux demandes de charlène }\end{array}$ \\
\hline $\begin{array}{l}\text { Patron « marqueur concessif }+ \\
\text { nous » } \\
\text { Conn CONC }+*+\text { nous }+ \\
\text { prédicat } \\
\text { (sentiment/pensée/constat) }\end{array}$ & $\begin{array}{l}\text { il dit qu'il souhaite que sa présence ne soit pas } \\
\text { facteur de perturbations pour didier mais nous } \\
\text { sentons dans ses propos un souhait de } \\
\text { réparation de la situation familiale compliquée } \\
\text { qu'il a dî vivre } \\
\text { toutefois, nous pensons qu'il y a lieu d'être } \\
\text { vigilant aux nouvelles demandes de sa famille } \\
\text { toutefois, nous constatons que toute } \\
\text { proposition venant d'un adulte lui demande } \\
\text { d'en vérifier le bien fondé }\end{array}$ \\
\hline $\begin{array}{l}\text { Patron « marqueur concessif }+ \\
\text { nous }+ \text { predicat sentiment } \\
\text { CONN CONC }+ \text { nous }+ \text { predicat } \\
\text { sentiment (peur/inquiétude) }\end{array}$ & $\begin{array}{l}\text { en revanche nous restons inquiets en ce qui } \\
\text { concerne les relations amoureuses de Jade } \\
\text { le collège parle de possibilité d'intégrer un } \\
\text { lycée professionnel standard mais nous } \\
\text { craignons que adèle ne se retrouve en décalage } \\
\text { important sur le plan scolaire } \\
\text { nous restons toutefois attentifs à l'évolution } \\
\text { d'Elise dans la mesure où elle présente un } \\
\text { retard de langage important }\end{array}$ \\
\hline
\end{tabular}

Tableau 2. Inventaire des patrons

Ce tableau appelle plusieurs remarques. Tout d'abord un patron peut produire des réalisations qui sont des expressions lexicalisées (phraséologie au sens restreint du terme) et des réalisations plus libres propres au corpus. On a donc une sorte de «moule » phraséologique qui est ici support de créations inédites. Ainsi pour les patrons « être dans » et « être en » :

être en difficulté, en conflit vs T est en recherche affective de la part de sa mère

être dans l'attente vs A est dans la dissimulation de ses devoirs ou mots à faire signer

Ensuite, certains patrons peuvent présenter des «sous-types ». Par exemple, une spécification du patron « être en » donne lieu à un deuxième patron $X$ est en difficulté pour : 


\section{$X$ est en/dans $+N$ prédicatif abstrait $\rightarrow X$ est en difficulté pour + SINF}

Ces différents degrés de figement participent à la texture des écrits tout en préservant la variété de l'expression (combinaisons différentes des patrons + combinatoire lexicale variée de chacun d'entre eux). C'est sans doute pour cette raison que les textes ne sont pas répétitifs - il ne s'agit pas de formulaires administratifs - tout en présentant entre eux un « air de famille ». Il va de soi que plus le patron est figé lexicalement, moins on relève d'occurrences.

Enfin, les patrons peuvent s'enchaîner, comme on le voit dans l'exemple suivant :

elle est par ailleurs, en grande demande affective, et peut être en retour très affectueuse et exprimer son attachement à sa famille d'accueil (X est en/dans N + ET PEUT)

Par ailleurs, nous allons montrer que ces patrons peuvent être mis en relation avec différentes déterminations discursives.

\section{Interprétation en fonction de différents niveaux de cotexte et contexte}

Dans une perspective d'analyse de discours, nous avons mis en relation les patrons identifiés avec des fonctionnements discursifs ainsi qu'avec des déterminations discursives et interdiscursives telles que la visée pragmatique, le rapport à l'allocutaire ou l'hétérogénéité discursive, voir infra, Tableau 3). Nous avons ainsi constaté que des patrons différents pouvaient participer d'un même fonctionnement discursif, et qu'un même patron pouvait être relié à plusieurs déterminations. Par ailleurs, utilisant les fonctions «topologiques» du Trameur, telle que la carte des sections, nous avons pu montrer comment un fonctionnement discursif pouvait s'actualiser plus particulièrement dans une «partie» du texte, participant ainsi à sa construction. Ces différents aspects nous ont conduites à la notion de « routines" que nous proposons de définir de la façon suivante :

Une routine discursive consiste en la mise en relation de séquences linguistiques récurrentes, partiellement figées (i.e. les patrons définis plus haut) avec des déterminations discursives et des fonctions textuelles propres à un genre ou une sphère d'activité.

Ces routines peuvent être envisagées comme des ressources fabriquées par et à disposition des scripteurs facilitant le processus d'écriture et participant de la construction d'une identité professionnelle tout en révélant des représentations propres au secteur. En effet, la focalisation sur le processus rédactionnel amène à prendre en compte les contraintes qui pèsent sur le processus d'écriture que l'on peut interpréter comme les contraintes exercées par le «texte déjà là ». On rejoint ici S. Plane et alii (2010:18-19) selon qui « le texte tel qu'il peut être saisi à un moment donné s'ancre toujours dans un texte préalable ». Pour ces auteurs, ce préalable dans la textualisation est de deux ordres : "les phénomènes relevant de représentations collectives, de doxas culturelles ou d'ordre socio-discursifs » et «[1]es phénomènes d'ordre structurel grâce auxquels la langue met à disposition du scripteur des configurations lexicales et syntaxiques déjà constituées » (figements, collocations).

Le tableau suivant présente un premier relevé des routines discursives mises au jour par l'analyse.

\begin{tabular}{|l|l|}
\hline Fonctionnement discursif & Patrons \\
\hline lieu commun du progrès & Patron « Temporalité - modalité (capacité) » \\
& ex: désormais il est capable de \\
& Patron « Pouvoir » + Prédicat « difficile » \\
& ex: il a pu nous faire part de ses réticences \\
& patron « et peut » \\
& ex: elle est autonome dans les jeux, et peut rester un long \\
\hline
\end{tabular}




\begin{tabular}{|c|c|}
\hline & moment à jouer seule dans sa chambre \\
\hline $\begin{array}{l}\text { donner la preuve (argument par } \\
\text { l'exemple) }\end{array}$ & $\begin{array}{l}\text { Patron « et peut" } \\
\text { ex: elle est autonome dans les jeux, et peut rester un long } \\
\text { moment à jouer seule dans sa chambre }\end{array}$ \\
\hline prendre position & $\begin{array}{l}\text { Patron « marqueur concessif + nous » } \\
\text { ex: toutefois, nous constatons que toute proposition venant } \\
\text { d'un adulte lui demande d'en vérifier le bien fondé } \\
\text { Patron « marqueur concessif }+ \text { nous }+ \text { prédicat } \\
\text { sentiment" } \\
\text { ex : en revanche nous restons inquiets en ce qui concerne les } \\
\text { relations amoureuses de Jade }\end{array}$ \\
\hline qualifier les personnes & $\begin{array}{l}\text { Patron « être dans" } \\
\text { ex: il est dans la dissimulation de ses devoirs ou mots à faire } \\
\text { signer } \\
\text { Patron « être en difficulté » } \\
\text { ex: madame est en grande difficulté pour se mobiliser autour } \\
\text { des besoins de ses enfants et de l'exercice de son autorité } \\
\text { parentale }\end{array}$ \\
\hline
\end{tabular}

Tableau 3. Inventaire provisoire des routines discursives

Afin d'illustrer les fonctions textuelles et discursives qui peuvent être assumées par les patrons, nous nous appuierons sur deux exemples : le cas du patron TEMPORALITE - MODALITE et le couple de patrons « X est dans »/ «X est en ».

\subsection{Le patron TEMPORALITE-MODALITE, expression du lieu commun du progrès}

La forme développée de ce patron est: ADV de temps (aspect imperfectif, rupture avec le passé) + prédicat exprimant la capacité. Un énoncé type de ce patron est : " désormais il/elle peut faire X », mais nous avons recensé les variantes combinatoires suivantes:

\begin{tabular}{|l|l|l|}
\hline désormais & il / elle & (est) capable de \\
aujourd'hui & & $\begin{array}{l}\text { démontre des capacités de } \\
\text { semble en capacité }\end{array}$ \\
beaucoup plus (= plus qu'avant) & & $\begin{array}{l}\text { a accès à } \\
\text { peut }\end{array}$ \\
maintenant & & peu de temps \\
plus qu'autrefois & & lui permettent de \\
\cline { 2 - 3 } & ses attitudes & \\
\hline
\end{tabular}

Tableau 4. Combinatoire du patron « Temporalité - modalité »

Ainsi que quelques exemples :

- «Pour autant, Didier démontre désormais certaines capacités d'autonomie qu'il a récemment acquises. » 
« Fabrice a nécessairement besoin de contenance et il peut maintenant questionner l'adulte [...]».

Certains connecteurs sont plus fréquemment associés que d'autres au patron. Si l'on prend les connecteurs temporels les plus fréquents, c'est le cas de désormais par exemple dans 8 cas (sur 11 occurrences), il participe à actualiser le patron, contre 14/30 pour aujourd'hui, et seulement 2 sur 8 pour maintenant (dans le SR peut maintenant). Cette attraction entre le patron et certains adverbes de temps nous a fait envisager dans un premier temps une description en termes de colligation (Sinclair 2004), c'est-à-dire la cooccurrence entre un item lexical ou grammatical et une catégorie grammaticale (partie du discours, fonction syntaxique...). Toutefois, la relation de colligation est asymétrique (organisée autour d'un mot noyau) : dans le cas qui nous occupe, quel serait le noyau central : les adverbes de temps? L'expression de la capacité ? Nous considérons que la configuration que nous décrivons est celle d'une unité composée : temporalité + modalité. C'est à ce niveau sémantico-pragmatique des notions que le patron trouve à exprimer son unité.

Le rapport avec le patron «POUVOIR » est sémantique : le prédicat (verbal ou adjectival) exprime la capacité. Ce sous-type du patron POUVOIR participe à l'expression du topos du progrès. La notion d'évolution est centrale au processus d'évaluation mis en œuvre par les éducateurs spécialisés dans leur pratique quotidienne. Elle est d'ailleurs centrale à d'autres processus évaluatifs, l'enseignement par exemple. La représentation professionnelle sous-jacente est celle que l'individu, enfant, jeune garçon ou jeune fille, qui leur est confié et dont ils vont s'occuper quotidiennement au foyer, a un " problème, une difficulté, est dans une situation délicate ou de danger » et qu'ils vont devoir régler ce problème, cette difficulté. Ce «règlement» nécessite d'évoluer pour au minimum changer mais surtout pour « améliorer».

Le patron en question articule progrès (les capacités) et expression d'une rupture par rapport à un état ancien de "problème », ce qui souligne le succès de l'intervention de l'éducateur pendant la période de placement de l'enfant, comme en témoigne sa présence dans les rubriques « évolution » et « conclusion » (voir figure 1).

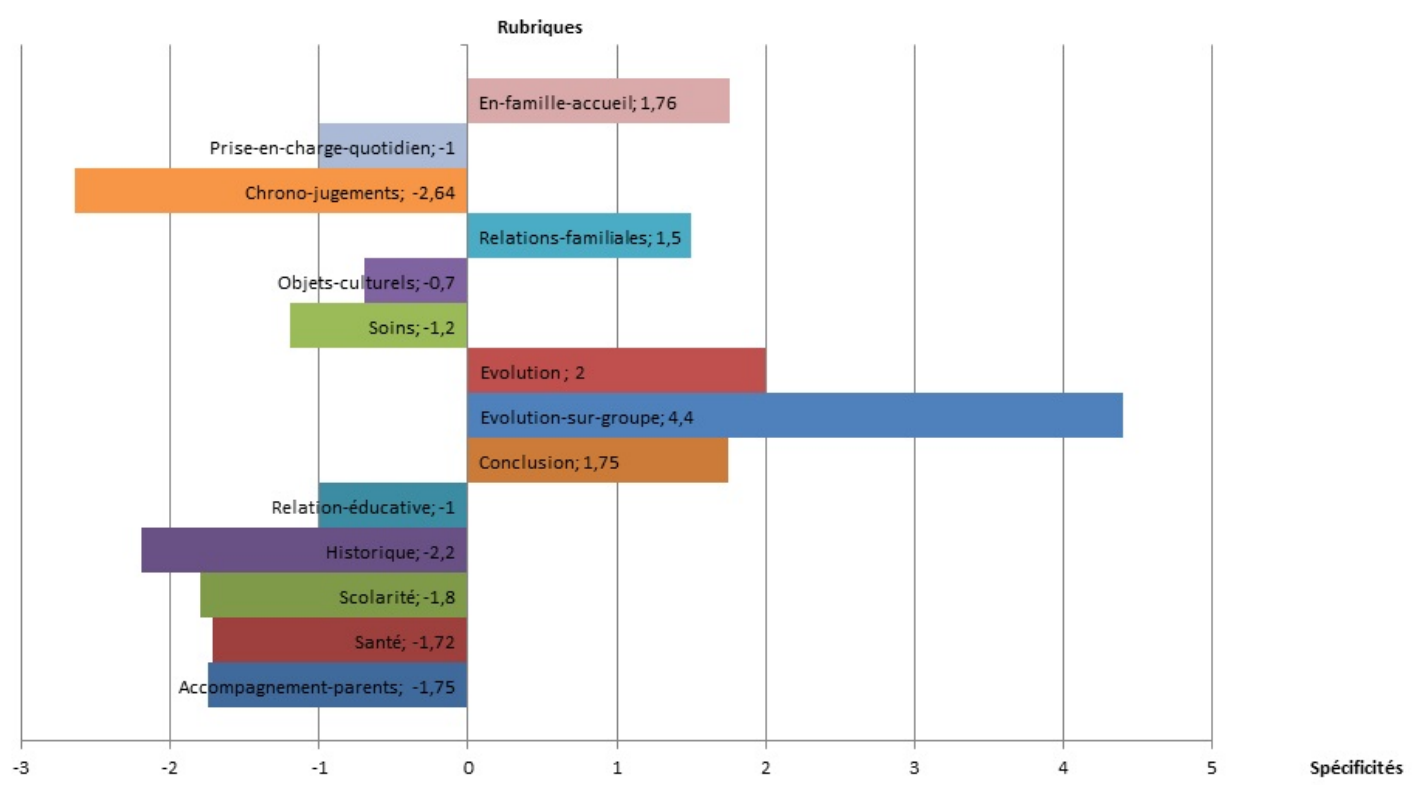

Figure 1. Spécificités des connecteurs temporels dans les rubriques (désormais, aujourd'hui, maintenant), Le Trameur

La distribution des patrons en fonction de la variable « rubrique $»^{2}$ permet de prendre en compte la surface du texte et de passer du discursif au textuel. Si l'on procède à une ventilation des connecteurs temporels par rubriques à travers un calcul des spécificités, on remarque une attirance pour certaines 
rubriques : « évolution », « conclusion », « évolution sur le groupe », « relation familiale », « en famille d'accueil ». L'interaction temporalité / modalité, avec les restrictions sémantiques précisées plus haut, est donc une association centrale dans l'écriture des éducateurs : en témoigne sa présence dans la conclusion et dans des rubriques qui sont au fondement de la pratique professionnelle des éducateurs ("évolution », " évolution sur le groupe »). D'autres marqueurs et notions participent aussi à l'expression du lieu commun, la comparaison par exemple, ou l'intensité.

\subsection{X est dans/X est en : différents niveaux de détermination discursive}

Les différentes déterminations et les niveaux de cristallisation s'articulent dans la production écrite. Nous pouvons tenter de déplier les différents niveaux de détermination à l'œuvre dans l'emploi fréquent des patrons $X$ est dans/en. D'une façon générale, ces patrons opèrent la caractérisation du $\mathrm{X}$ en position sujet, qui désigne dans la majorité des cas l'enfant ou sa famille, "objets » du rapport. Ils s'inscrivent donc dans la visée de caractérisation/évaluation à laquelle répond le rapport éducatif, et qui sert de support à la préconisation. Si l'on compare avec l'effet que produirait l'emploi du verbe simple correspondant :

Il peut être ensuite [après avoir cassé] dans la réparation vs il peut réparer (quand il a cassé quelque chose)

Il est dans la dissimulation de ses devoirs vs Il dissimule ses devoirs

Sa mère avait été en total soutien à son beau-père vs Sa mère avait totalement soutenu son beau-père

On peut constater que le verbe simple caractérise le sujet en racontant ou en décrivant ce qu'il fait et qu'employé au présent, il peut prendre une valeur de vérité générale (il dissimule ses devoirs).

La tournure avec dans en revanche procède à une caractérisation de la personnalité de $\mathrm{X}$ en le situant par rapport à un état psychologique ou à un affect (il est dans la dissimulation, dans l'évitement). Le $\mathrm{N}$ abstrait dans cette tournure est interprétable comme dénotant un état psychologique. Le sujet n'est plus acteur, il est d'une certaine façon « agi », déresponsabilisé.

L'effet produit par être en est différent : il marque au contraire une « sorte de solidarité, facilement active, au contraire, en tout cas strictement indissociable entre le sujet et son activité »(J.-J. Franckel, communication personnelle). De fait les réalisations de $X$ est en sont plus proches d'expressions lexicalisées (être en colère, être en difficulté), tandis que la créativité/la variété des réalisations est plus importante avec $X$ est dans (voir Tableau 2).

Pour les professionnels, interrogés sur la question lors d'une restitution, l'emploi de cette tournure est ressenti comme «moins négatif » que celle du verbe simple équivalent dans la mesure où elle marque le caractère transitoire de l'évaluation. La routine est donc ici pour une part consciente et peut faire l'objet d'une verbalisation.

Mais on ne peut ignorer que la façon dont l'expression il est dans en particulier opère la caractérisation du sujet peut relever d'un regard clinique «surplombant». Nos travaux nous ont permis de constater à plusieurs reprises la porosité du discours des travailleurs sociaux avec ce que nous avons appelé le « discours psy» (psychologique/psychanalytique). Il semble cependant difficile de parler ici d'emprunt au sens propre du terme, étant donné la diversité et le caractère créatif des tournures - à moins qu'elles ne soient reprises au discours tenu par le psychologue du service lors des réunions de synthèse. Nous dirions plutôt que ces expressions « résonnent» comme une « façon de dire » propre au discours psy dont elles « imitent » et font d'une certaine façon proliférer les patrons caractéristiques. Une description de ces deux patrons ne peut faire l'économie de ces deux niveaux de déterminations : la fonction de caractérisation de la personne et l'interdiscours «psy ». 


\section{Conclusion}

Notre travail s'inscrit très clairement dans l'extension du domaine de la phraséologie, ce qui nous a permis de mettre au jour des séquences multidimensionnelles articulant lexique, syntaxe, texte, discours et pragmatique. Nos patrons sont certainement moins figés que d'autres unités des recherches phraséologiques. Ils se construisent le plus souvent autour d'un noyau stable qui s'articule à des variantes combinatoires dont le paradigme peut être mis en relation avec des notions sémantico-grammaticales (temporalité, modalité ou difficulté). Parmi les résultats notables de cette étude, nous souhaitons mettre l'accent sur l'interaction du texte et du discours. En effet, l'observation du fonctionnement discursif des patrons permet l'accès à des fonctionnements textuels par la prise en compte des rubriques, c'est-à-dire des «lieux » et de la structure du texte où apparaissent les patrons. La notion de routine discursive est apparue productive sur un corpus de ce type. Des questions demeurent en suspens : 1) celle du niveau de cristallisation des routines : le scripteur, le genre, la sphère d'activité, le service ? 2) celle du rôle de facilitateur d'écriture que nous avons postulé pour ces routines. Un des objectifs de notre projet de recherche est de mettre en relation les routines observées sur les états finaux avec les données enregistrées en temps réel, ce qui permettra de proposer des éléments de réponse à cette question. On pourrait également envisager un retour réflexif des éducateurs sur les routines dégagées par notre analyse.

\section{Bibliographie}

Bolly, C. (2011). Phraséologie et collocations. Berne : Peter Lang.

Branca-Rosoff, S. (1990). « Conventions d'écriture dans la correspondance des soldats ». Mots, 24, 21-36.

Branca-Rosoff, S. (1997). " Modèles de locutionnarité et effets de figement dans le discours politique de l'an II ». In Fiala, P., Lafon, P., Piguet, M.-F. (éds.), La locution: entre lexique, syntaxe et pragmatique, Paris : Klincksieck, 285- 293.

Charaudeau, P. (2001). «Visées discursives, genres situationnels et construction textuelle ». In Ballabriga, M. (Ed), Analyse des discours, Types et genres: communication et interprétation, Toulouse : Ed. Universitaires du Sud, [http://www.patrick-charaudeau.com/Visees-discursives-genres, 83.html].

Cislaru, G., Sitri, F., Pugnière-Saavedra, F. (à paraitre), «Figement et configuration textuelle : les segments de discours répétés dans les rapports éducatifs", actes du colloque LPTS 2011 "Variation(s) sur la structure de l'oral et l'écrit », à paraître dans Corpora and Language in use.

Cislaru, G., Pugnière, F., Sitri, F., Veniard, M. (éds.) (2008). Les Carnets du Cediscor, 10, Analyse de discours et demande sociale : le cas des écrits de signalement.

Fiala, P., Habert, B., Lafon P., Pineira, C. (1987). «Des mots aux syntagmes, figements et variations dans la Résolution générale du congrès de la CGT de 1978 ». Mots, 14, 45-87.

Fiala, P. (1987). « Pour une approche discursive de la phraséologie. Remarques en vrac sur la locutionnalité et quelques points de vue qui s'y rapportent, sans doute ». Langage et Société, 42, 28-48.

Fleury, S. (2007). Le Métier Textométrique: Le Trameur, Manuel d'utilisation. Université Paris 3, Centre de Textométrie. http://tal.univ-paris3.fr/trameur/

Heiden, S., Lafon, P. (1998). «Cooccurrences. La CFDT de 1973 à 1992 ». In Des mots en liberté, Mélanges Maurice Tournier, Paris : ENS Éditions, tome 1, 65-83.

Krieg-Planque, A. (2008). «La notion d'observable en discours. Jusqu'où aller avec les sciences du langage dans l'étude des pratiques d'écriture journalistique ?». In Marcel Burger (dir.), L'analyse linguistique des discours médiatiques. Entre sciences du langage et sciences de la communication, Québec, Université de Laval, Editions Nota Bene, 59-81.

Lafon, P. (1981). « Analyse lexicométrique et recherche des cooccurrences ». Mots, 3, 95-148.

Longrée, D., Luong, X. et Mellet, S. (2008). «Les motifs : un outil pour la caractérisation topologique des textes. » In S. Heiden et B. Pincemin (éds), JADT 2008, Actes des 9èmes Journées internationales d'Analyse statistique des Données Textuelles, vol. 2, Lyon: Presses universitaires de Lyon: 733-744. [En ligne]: http://lexicometrica.univ-paris3.fr/jadt/jadt2008/pdf/Longréeluong-mellet.pdf 
Longrée, D., Mellet, S. (2013). «Le motif: une unité phraséologique englobante ? Étendre le champ de la phraséologie de la langue au discours ». Langages, 189, 65-79.

Maingueneau, D. (1991). L'analyse du discours. Introduction aux lectures de l'archive. Paris : Hachette Université.

Maingueneau, D. (2004). « Retour sur une catégorie : le genre ». In Adam, J.-M., Grize J.-B. et Ali Bouacha M. (eds), Texte et discours, catégories pour l'analyse, Dijon : Editions Universitaires de Dijon, 107-118.

Martinez, W. (2003). Contribution à une méthodologie de l'analyse des cooccurrences lexicales multiples dans les corpus textuels. Thèse pour le doctorat en Sciences du Langage, Université Sorbonne nouvelle - Paris 3.

Mayaffre, D. (2008). «Quand «travail », « famille », «patrie » co-occurrent dans le discours de Nicolas Sarkozy. Étude de cas et réflexion théorique sur la co-occurrence ». In S. Heiden et B. Pincemin (éds), JADT 2008, Actes des 9èmes Journées internationales d'Analyse statistique des Données Textuelles, vol. 2, Lyon: Presses universitaires de Lyon, 811-822. [En ligne] http://lexicometrica.univ-paris3.fr/jadt/jadt2008/pdf/mayaffre.pdf

Née, E., MacMurray, E., Fleury, S. (2012), « Textometric Explorations of writing processes: a discursive and genetic approach to the study of drafts", Actes des Journées internationales d'Analyses statistiques des Données Textuelles (JADT 2012), Liège, 13-15 juin 2012, [En ligne] http://lexicometrica.univparis3.fr/jadt/jadt2012/Communications/Nee, $\% 20$ Emilie $\% 20$ et $\% 20 \mathrm{al} . \% 20$ $\% 20$ Textometric $\% 20$ Explorations $\% 20 \mathrm{of} \% 20$ Writing $\% 20$ Processes.pdf.

Plane, S., Alarmagot, D., Lebrave, J.-L. (2010). «Temporalité de l'écriture et rôle du texte produit dans l'activité rédactionnelle ». Langages, 177, 7-28.

Poudat, C. (2006). Etude contrastive de l'article scientifique de revue linguistique dans une perspective d'analyse des genres. Thèse pour le doctorat en Sciences du Langage, Université d'Orléans.

Salem, A. (1987). Pratique des segments répétés. Essai de statistique textuelle. Paris : Klincksieck.

Sinclair, J., Carter, R. (scientific editor) (2004). Trust the text. Language, corpus and discourse, Londres : Routledge.

Sitri, F., 2013. «Une lecture évènementielle du verbe pouvoir dans des rapports de travailleurs sociaux ». In Moirand, S., Reboul-Touré S., Londei D., Reggiani L. (éds.), Dire l'évènement. Langage, mémoire, société, Paris : Presses de la Sorbonne nouvelle, p. 73-83.

Sitri, F., 2008, «Observer et évaluer dans les rapports éducatifs : de la représentation d'un dire singulier à la description d'une situation », Les Carnets du Cediscor, 10, 95-116.

Tutin A., Grossmann F. (2002). "Collocations régulières et irrégulières : esquisse de typologie du phénomène collocatif ». Revue Française de Linguistique Appliquée, Lexique : recherches actuelles, vol VII, 7-25. (Version pdf : http://w3.u-grenoble3.fr/tutin/Publis/RFLA.pdf)

Tutin, A. (2007). " Autour du lexique et de la phraséologie des écrits scientifiques », Revue française de linguistique appliquée, vol. XII, 5-14.

Veniard, M. (2008). «Écrire 'ce qui ne va pas' dans le champ de l'enfance en danger: les mots problème(s) et difficulté(s) », Les carnets du Cediscor, 10, 57-77.

\footnotetext{
${ }^{1}$ On pourrait discuter dans le détail la caractérisation du discours syndical comme « genre », dans la mesure où, en fonction de l'approche qui est la nôtre, nous établirions une différence entre le genre de la résolution de congrès et la sphère syndicale. Il n'en reste pas moins que notre objectif ici est bien, à partir d'une analyse qualitative des segments et patrons repérés, de tenter de « rendre compte » de leur figement et de leur constitution en routines.

${ }^{2}$ La variable « rubrique » tient compte des rubriques telles qu'elles apparaissent dans ces rapports éducatifs.
} 